\title{
Papers
}

\section{Adenoidectomy versus chemoprophylaxis and placebo for recurrent acute otitis media in children aged under 2 years: randomised controlled trial}

\author{
Petri Koivunen, Matti Uhari, Jukka Luotonen, Aila Kristo, Risto Raski, Tytti Pokka, Olli-Pekka Alho
}

\begin{abstract}
Objective To evaluate the efficacy of adenoidectomy compared with long term chemoprophylaxis and placebo in the prevention of recurrent acute otitis media in children aged between 10 months and 2 years.

Design Randomised, double blind, controlled trial. Setting Oulu University Hospital, a tertiary centre in Finland. Participants 180 children aged 10 months to 2 years with recurrent acute otitis media.

Intervention Adenoidectomy, sulfafurazole (sulphisoxazole) 50 $\mathrm{mg} / \mathrm{kg}$ body weight, given once a day for six months or placebo. Follow up lasted for two years, during which time all symptoms and episodes of acute otitis media were recorded.

Main outcome measures Intervention failure (two episodes in two months or three in six months or persistent effusion) during follow up, number of episodes of acute otitis media, number of visits to a doctor because of any infection, and antibiotic prescriptions. Number of prescriptions, and days with symptoms of respiratory infection.

Results Compared with placebo, interventions failed during both the first six months and the rest of the follow up period of 24 months similarly in the adenoidectomy and

chemoprophylaxis groups (at six months the differences in risk were $10 \%$ (95\% confidence interval $-9 \%$ to $29 \%)$ and $18 \%$ ( $-2 \%$ to $38 \%$ ), respectively). No significant differences were observed between the groups in the numbers of episodes of acute otitis media, visits to a doctor, antibiotic prescriptions, and days with symptoms of respiratory infection.

Conclusions Adenoidectomy, as the first surgical treatment of children aged 10 to 24 months with recurrent acute otitis media, is not effective in preventing further episodes. It cannot be recommended as the primary method of prophylaxis.
\end{abstract}

\section{Introduction}

About one third of all children experience recurrent episodes of acute otitis media. ${ }^{1-4}$ Although there are many preventive strategies, none seem to be indisputably effective. ${ }^{5-8}$ Adenoidectomy may benefit the middle ear by removing a source of infection from the nasopharynx ${ }^{5}$ and has been shown to be helpful in children over 4 years of age with chronic otitis media with effusion. ${ }^{9}{ }^{10}$ In contrast, there is much less evidence of the efficacy of adenoidectomy in preventing recurrent episodes of acute otitis media. Paradise et al found that adenoidectomy, as the first surgical intervention, did not have a significant effect on recurrent episodes, ${ }^{11}$ but among children previously treated with tympanostomy tubes it reduced further attacks. ${ }^{12}$ Both of these trials involved children over 3 years of age, and there is virtually no empirical evidence on the effectiveness of adenoidectomy as the first surgical treatment in preventing recurrent acute otitis media in children aged under 2 years, who are clearly at the highest risk.

The short term objectives in otitis media prophylaxis are to reduce pain, fever, parental anxiety, and costs. With this in mind, we assessed the usefulness of adenoidectomy in preventing further acute episodes, relieving acute symptoms, and reducing the numbers of visits to a doctor because of any infection and prescriptions for antibiotics compared with chemoprophylaxis and placebo in a randomised controlled trial on children aged under 2 years with recurrent acute otitis media.

\section{Methods}

\section{Enrolment and assignment}

We selected participants from all children aged 10-24 months who were referred to the department of otolaryngology at Oulu University Hospital for recurrent acute otitis media from 1 April 1994 to 17 April 1997. To be eligible, the child had to have experienced at least three acute episodes during the previous six months. Exclusion criteria were a previously performed adenoidectomy or tympanostomy, cranial anomalies, documented immunological disorders, and ongoing antimicrobial chemoprophylaxis.

We obtained background data and informed consent from parents. The children were examined at assignment by a validated otoscopist, ${ }^{13}$ and if middle ear effusion was detected, amoxicillin was prescribed. If effusion was still found two weeks later, myringotomy was performed. Effusion was monitored for two months. If effusion was still present after two months a tympanostomy tube was inserted and the assigned treatment given.

\section{Intervention}

The adenoidectomy operation was performed as daycare surgery. Chemoprophylaxis comprised sulfafurazole (sulphisoxazole) suspension $50 \mathrm{mg} / \mathrm{kg}$ of body weight, given once a day for six months. The placebo was given at a similar frequency and volume.

\section{Follow up}

The children were followed up for two years by means of symptom diaries and clinical examinations from the first day without effusion. We recorded all acute symptoms, episodes of acute otitis media, and visits to a doctor. Control visits to one of the three study otolaryngologists were scheduled at least every four 


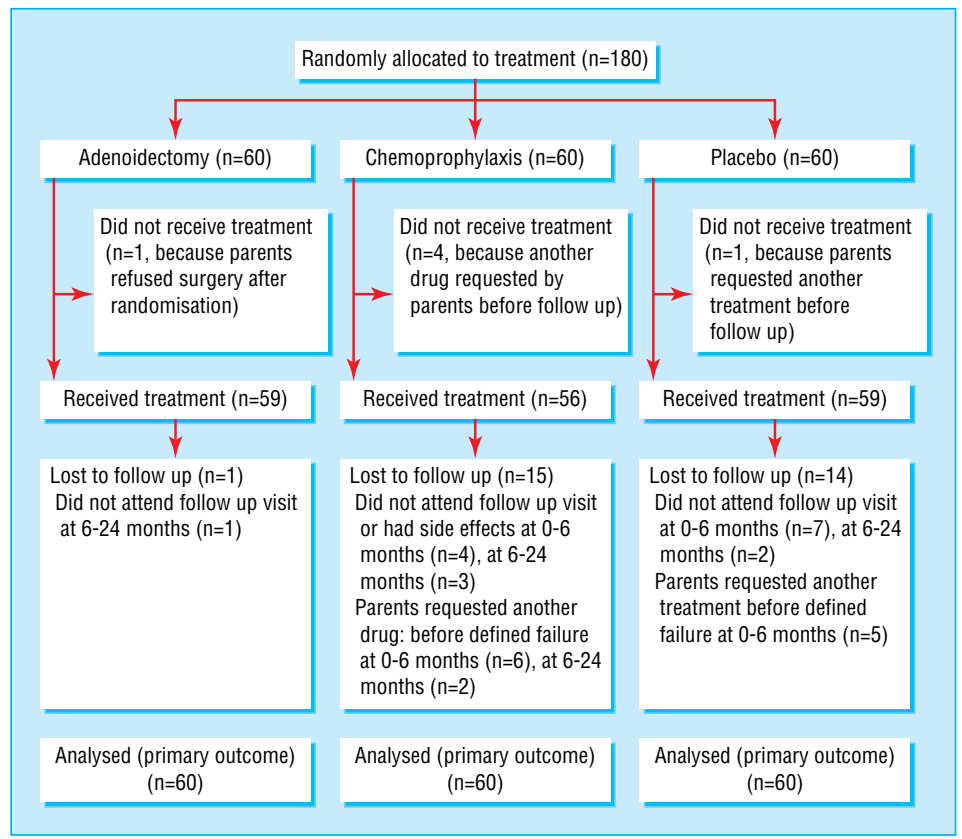

Fig 1 Trial profile and participant flow

months with an assessment of ear status and compliance and collection of the symptom diaries.

Whenever the child had acute respiratory symptoms, the parents were advised to visit their own general practitioner or the study otolaryngologists. The criteria for acute otitis media consisted of acute symptoms together with signs of middle ear inflammation (hyperaemic, opaque, or bulging ear drum) and middle ear effusion obtained in pneumatic otoscopy or otorrhoea. Each acute episode was managed with an antimicrobial drug, usually amoxicillin for one week, and a control visit was scheduled with the study otolaryngologist within two weeks. In the case of a prolonged episode, another antibiotic microbial was prescribed and control visits were scheduled every two weeks until the middle ear was found to be free from effusion.

\section{Intervention failure}

Intervention was deemed to have failed whenever the child experienced two acute episodes in two months or three episodes in six months based on the diary, or if the child had middle ear effusion for at least two months as assessed by the study otolaryngologist using a pneumatic otoscope.

\section{Outcome measures}

The primary outcome measure was intervention failure during the first six months of follow up. The secondary outcome measures were mean numbers of episodes of acute otitis media, visits to a doctor, antibiotic prescriptions, days of symptoms (rhinitis, earache, fever), and adverse effects as recorded in the diary.

\section{Sample size and data analysis}

Our primary aim was to compare the adenoidectomy and placebo groups. On the basis of our experience, ${ }^{14}$ we expected a $50 \%$ failure rate in the placebo group. A 25 percentage point decrease in this rate in the adenoidectomy group was considered clinically significant. Based on two tailed testing with $\alpha=0.05$ and $\beta=0.20$ we estimated that 57 children were needed per group. ${ }^{15}$ To reach this number, we recruited 60 children in each group.

The failure percentages were calculated with the KaplanMeier method, where the cases lost to follow up are included until the dropout date. We compared the groups by calculating the differences in these percentages with $95 \%$ confidence intervals using the confidence interval analysis software. ${ }^{16}$ The times to the intervention failure were presented by means of Kaplan-Meier curves, and the differences between the groups were tested with the log rank test. To compare the mean numbers of episodes of acute otitis media, visits to a doctor, antibiotic prescriptions, and days of symptoms we calculated differences in means (95\% confidence intervals).

We regarded those children who did not get the allocated prophylaxis or whose prophylaxis was changed before defined failure as protocol violations. The fact that the number of protocol violations was higher in the non-surgical arms than in the surgical arm made the interpretation of the data more difficult (see fig 1). We do not know the reasons for this dissimilar parental behaviour between the groups, but the possibility of discontinuation is not equal between the groups as one cannot discontinue adenoidectomy. To allow several possible interpretations, we analysed the data by regarding the protocol violations as both drop outs and failures.

\section{Assignment and blinding}

We randomly assigned children to receive adenoidectomy or six months' prophylaxis with sulfafurazole or placebo suspension of the same colour and taste. We performed block randomisation with a block size of six using numbered containers so that allocation was concealed from the investigator. The sulfafurazole and placebo suspensions were given in a double blind fashion.

\section{Results}

Follow up of participants-Altogether 180 children were randomly allocated to one of the treatment groups (table 1, fig 1). Children in the sulfafurazole and placebo groups discontinued intervention and received another prophylaxis more often than children in the adenoidectomy group (fig 1). Twelve children in the adenoidectomy group underwent concurrent tympanostomy because secretory middle ear fluid was found at the operation. 
Table 1 Baseline characteristics of 180 children with recurrent acute otitis media randomised to receive adenoidectomy, sulfafurazole prophylaxis, or placebo. Figures are numbers of children unless stated otherwise

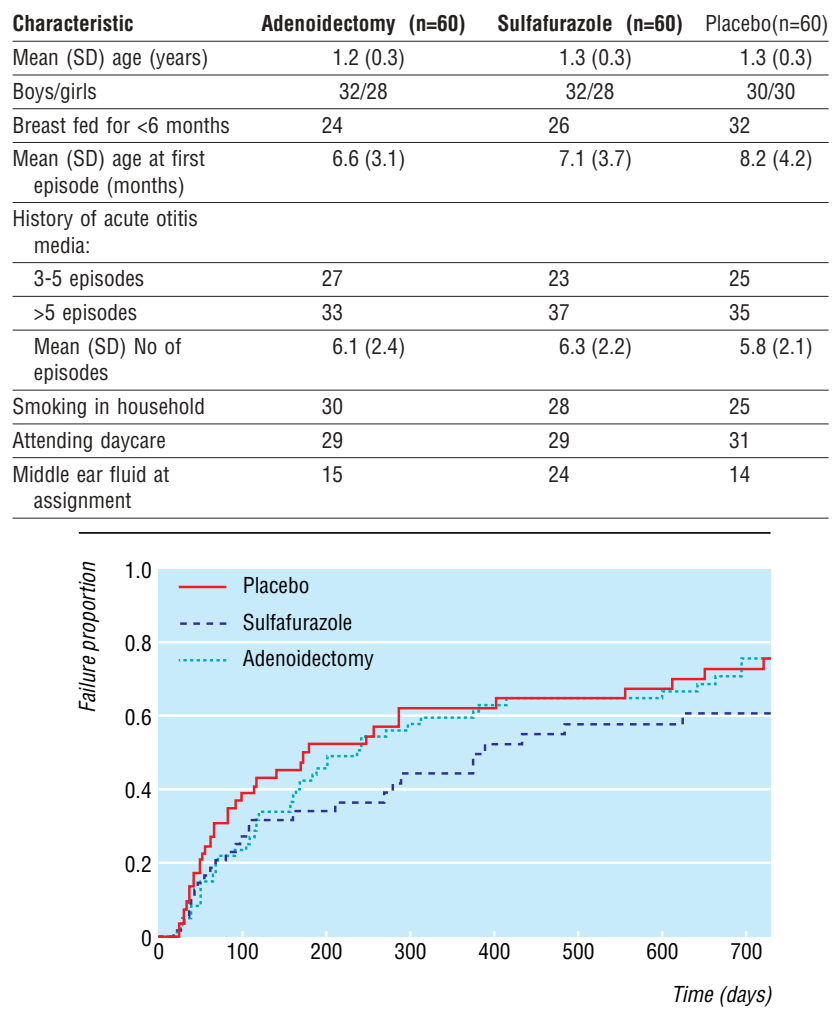

Fig 2 Cumulative occurrence of failures during two year follow up in 180 children, by treatment groups (adenoidectomy, chemoprophylaxis, placebo). Failure recorded if child had two episodes of acute otitis media within two months or three episodes within six months or middle ear effusion that persisted for two months. Protocol violations regarded as drop outs. No significant differences in time to failure between groups ( $P=0.22$ at 6 months; $P=0.28$ at 24 months, log rank test)
Outcome at six months-Intervention failed in 25 children in the adenoidectomy group (one drop out) and in 26 in the placebo group (13 drop outs) (difference in percentage failure $10 \%, 95 \%$ confidence interval $-9 \%$ to $29 \%$; protocol violations regarded as drop outs) (table 2). The figures when we counted protocol violation as failure were 26 in the adenoidectomy group (no drop outs) and 32 in the placebo group (seven drop outs) $(15 \%,-3 \%$ to $33 \%)$ (table 2). Intervention failed in 17 children in the sulfafurazole group (14 drop outs), showing a 18\% (-2\% to $38 \%$ ) decrease in risk compared with the placebo group. There were no significant differences between the groups in the time to intervention failure (fig 2). There were no significant differences between the groups in the numbers of episodes of acute otitis media, visits to a doctor, antibiotic prescriptions, and days with symptoms of respiratory infection (table 3).

Outcome at 24 months-At 24 months treatment failure was similar in the three groups (table 2). The number of children who needed tympanostomy tubes because of persistent middle ear fluid was somewhat lower in the adenoidectomy and sulfafurazole groups than in the placebo group $(6,6$, and 11 children, respectively).

Adverse effects-There were no complications in the adenoidectomy procedures (no serious haemorrhage, fever, or persistent emesis). Five children in the sulfafurazole group (two had diarrhoea, two had skin rashes, one unknown) and two children in the placebo group (one had diarrhoea, one unknown) were reported to have adverse effects.

Compliance-The mean numbers of forgotten doses in the chemoprophylaxis and placebo groups during the six months were 4.5 (range 3-100) and 2.2 (1-17), respectively. This difference was due to one child who was given sulfafurazole irregularly.

\section{Discussion}

Adenoidectomy as the primary treatment for recurrent acute otitis media in children aged under 2 years slightly diminished the risk of further acute recurrences and of persistent middle ear

Table 2 Main outcome measures in children with recurrent acute otitis media randomised to receive adenoidectomy, sulfafurazole prophylaxis, or placebo

\begin{tabular}{|c|c|c|c|c|c|c|c|c|}
\hline \multirow[b]{2}{*}{ Time } & \multicolumn{3}{|c|}{ Adenoidectomy } & \multicolumn{3}{|c|}{ Sulfafurazole } & \multicolumn{2}{|c|}{ Placebo } \\
\hline & $\mathrm{No}^{*}$ & $\begin{array}{l}\text { No }(\%) \text { of } \\
\text { failures } \dagger\end{array}$ & Difference $(95 \%$ CI) & No* & $\begin{array}{l}\text { No }(\%) \text { of } \\
\text { failures } \dagger\end{array}$ & Difference $(95 \%$ CI) & No* & $\begin{array}{c}\text { No }(\%) \text { of } \\
\text { failures }\end{array}$ \\
\hline \multicolumn{9}{|c|}{ Analysis where protocol violations were regarded as drop outs } \\
\hline At 6 months & $59 / 60$ & $25(42)$ & 10 (-9 to 29) & $46 / 60$ & $17(34)$ & $18(-2$ to 38$)$ & $47 / 60$ & $26(52)$ \\
\hline At 24 months & $58 / 60$ & $42(76)$ & $0(-17$ to 17$)$ & $41 / 60$ & $27(60)$ & $15(-4$ to 35$)$ & $45 / 60$ & $35(76)$ \\
\hline \multicolumn{9}{|c|}{ Analysis where protocol violations were regarded as failures } \\
\hline At 6 months & $60 / 60$ & $26(43)$ & 15 (-3 to 33$)$ & $56 / 60$ & $27(47)$ & $12(-7$ to 30$)$ & $53 / 60$ & $32(58)$ \\
\hline At 24 months & $59 / 60$ & $43(76)$ & $3(-13$ to 19$)$ & $53 / 60$ & $39(71)$ & 7 (-9 to 24$)$ & $51 / 60$ & $41(79)$ \\
\hline
\end{tabular}

`Effective sample size (total minus drop outs)/total sample size.

†Defined as having two episodes of acute otitis media in two months or three in six months or middle ear effusion for two months. ¥Compared with placebo.

Table 3 Secondary outcome measures in children with recurrent acute otitis media randomised to receive adenoidectomy, prophylaxis with sulfafurazole, or placebo*

\begin{tabular}{|c|c|c|c|c|c|c|c|}
\hline \multirow{3}{*}{$\begin{array}{l}\text { Measure } \\
\text { Episodes of acute otitis media }\end{array}$} & \multicolumn{3}{|c|}{ Adenoidectomy } & \multicolumn{3}{|c|}{ Sulfafurazole } & \multirow{2}{*}{$\begin{array}{l}\text { Mean (SD) on } \\
\text { placebo }\end{array}$} \\
\hline & Mean (SD) & \multicolumn{2}{|c|}{ Difference in mean† $(95 \% \mathrm{Cl})$} & \multirow{2}{*}{$\begin{array}{cc}\text { Mean } & \text { (SD) } \\
1.0 \quad(0.8)\end{array}$} & \multicolumn{2}{|c|}{ Difference in mean† $(95 \% \mathrm{Cl})$} & \\
\hline & $1.3 \quad(0.9)$ & 0.0 & $(-0.4$ to 0.4$)$ & & 0.3 & $(-0.1$ to 0.6$)$ & $1.3 \quad(0.9)$ \\
\hline Sick child visits & $3.3 \quad(1.8)$ & 0.6 & $(-0.3$ to 1.5$)$ & $3.7 \quad(2.0)$ & 0.2 & $(-0.7$ to 1.1$)$ & $3.9 \quad(2.4)$ \\
\hline Antimicrobial prescriptions & $1.5 \quad(1.1)$ & -0.1 & $(-0.6$ to 0.5$)$ & $1.4 \quad(1.3)$ & 0 & $(-0.5$ to 0.6$)$ & $1.4 \quad(1.2)$ \\
\hline Days with rhinitis & $26 \quad(23)$ & -4 & to 7 ) & $23 \quad(24)$ & 0 & 10) & $23 \quad(25)$ \\
\hline Days with earache & $1.9 \quad(3.1)$ & -0.3 & $(-1.6$ to 1.0$)$ & $1.7 \quad(3.4)$ & -0.1 & $(-1.4$ to 1.2$)$ & $1.6 \quad(2.4)$ \\
\hline Days with fever & $5.3 \quad(8.2)$ & -1.6 & (-4.5 to 1.2$)$ & $4.8 \quad(6.7)$ & -1.1 & (-3.9 to 1.8$)$ & $3.7 \quad(4.3)$ \\
\hline
\end{tabular}

${ }^{*}$ Analysed until intervention failure, drop out, or six months of follow up, whichever came first.

†Compared with placebo. 
effusion, but the beneficial effect, if any, seems to be so small that we cannot recommend it as the primary prophylactic method in this age group. The small differences between the groups seen during the first six months levelled off during the rest of the follow up. Adenoidectomy did not affect acute symptoms or the numbers of visits to a doctor and antimicrobial prescriptions.

The parents of a child prone to otitis usually have strong opinions about their child's preferred treatment, which makes it difficult to accept random allocation, even if they decide to participate in a trial. ${ }^{12} 18$ In our study, parental agreement to participate was good, and the number of children who did not receive the allocated intervention was quite small, but still some of the children in the chemoprophylaxis and placebo groups were given another prophylaxis in response to parental request or did not attend the follow up visits. The results did not change essentially even when we interpreted the protocol violations as failures. However, as the children in the placebo and sulfafurazole groups discontinued the allocated intervention without clinically defined failure more often than the children in the adenoidectomy group (in whom tympanostomy tubes could have been inserted or antibiotics also given), and as these children may have had more severe otitis media, this could have caused some bias by weakening the true effect of adenoidectomy. Twelve children in the adenoidectomy group received tympanostomy tubes because of secretory middle ear effusion, but we would expect the tubes to have improved the outcome rather than impaired it.

These results agree with the conclusions of Paradise et al on older children, which suggested that adenoidectomy should not be considered as a first surgical intervention in children with recurrent acute otitis media. ${ }^{11}$ Similarly, a recent study indicated that in children younger than 2 years, concurrent adenoidectomy with tympanostomy tubes did not have any major advantage over tubes alone in preventing otitis media. ${ }^{18}$ In contrast, among older children adenoidectomy has been found to be helpful, particularly in chronic otitis media. ${ }^{9} 101219$

We failed to show any significant effect of long term prophylaxis with sulfafurazole in recurrent otitis media. We do not know whether this lack of effect was due to the antimicrobial used because the evidence of differences in efficacy between antimicrobials is controversial. ${ }^{8}$ The worldwide crisis of multiple resistance and the poor compliance with non-surgical treatments further limit the usefulness of chemoprophylaxis.

There are two other important sources of bias that may have diminished the true effect of the treatments: firstly, the use of symptom diaries to collect the outcome, and, secondly, the possibility of misdiagnoses. We reminded the parents about the importance of recording the events, and the symptom diaries were colourful leaflets, which were collected every four months. Most of the diagnoses of otitis were made by general practitioners working in the health centres of one city and four surrounding communities who have been trained to use a pneumatic otoscope. In addition, follow up visits with the investigating otolaryngologists were scheduled after the acute episodes and regularly every four months. The number of treatment failures in our study was high, which reflects the young age of the children and success in the enrolment of children at a high risk of otitis media. In view of the baseline characteristics, our results are generalisable to the population seen in primary care in Finland. ${ }^{20}$

\section{Conclusion}

Adenoidectomy, as the first surgical treatment for recurrent acute otitis media, is not effective in preventing further acute epi-

\section{What is already known on this topic}

Adenoidectomy may affect the middle ear beneficially by removing of a source of infection from the nasopharynx and is often used to prevent recurrences of otitis media

Little is known about the effectiveness of adenoidectomy in preventing recurrent acute otitis media in children aged under 2 years, who are clearly at the highest risk

\section{What this study adds}

In this randomised controlled trial, compared with chemoprophylaxis and placebo adenoidectomy as the primary treatment for recurrent acute otitis media in children aged under 2 years did not significantly diminish the risk of acute recurrences

Adenoidectomy cannot be recommended as the primary method of prophylaxis for children aged under 2 years

sodes. We cannot recommended it as the primary method of prophylaxis for children aged under 2 years.

Contributors: PK planned the study, collected, analysed, and interpreted the data, and is guarantor. MU, JL, and O-PA designed the protocol and analysed and interpreted the data. AK and RR collected and interpreted the data. TP analysed the results. The manuscript was prepared by PK and $\mathrm{O}-\mathrm{PA}$ and commented on by all authors.

Funding: Departments of Paediatrics and Otorhinolaryngology, Oulu University Hospital.

Competing interests: None declared.

Ethical approval: The protocol was approved by the ethical committee of Oulu University Hospital.

1 Sipilä M, Pukander J, Karma P. Incidence of acute otitis media up to the age of 1 1/2 years in urban infants. Acta Otolaryngol 1987;104:138-45.

2 Teele DW, Klein JO, Rosner B, the Greater Boston Otitis Media Study Group. Epidemiology of otitis media during the first seven years of life in children in greater Boston: a ology of otitis media during the first seven years of life
prospective, cohort study. Jnfect Dis 1989;160:83-94.

3 prospective, cohort study. J Infect Dis 1989;160:83-94. Alho O-P. How
1997;529:8-10.

4 Lanphear BP, Byrd RS, Auinger P, Hall CB. Increasing prevalence of recurrent otitis media among children in the United States. Pediatrics 1997;99:e1-7.

5 Sade J, Luntz M. Adenoidectomy in otitis media-a review. Ann Otol Rhinol Laryngol 1991;100:226-31.

6 Casselbrant ML, Kaleida PH, Rockette HE, Paradise JL, Bluestone CD, Kurs-Lasky M. Efficacy of antimicrobial prophylaxis and of tympanostomy tube insertion for prevention of recurrent acute otitis media: results of a randomized clinical trial. Pediatr Infect Dis J 1992;11:278-86.

7 Mandel EM, Rockette HE, Bluestone CD, Paradise JL, Nozza RJ. Efficacy of myringotomy with and without tympanostomy tubes for chronic otitis media with effumyringotomy with and without tympan
sion. Pediatr Infect Dis J 1992;11:270-7.

8 Williams RL, Chalmers TC, Stange KC, Chalmers FT, Bowlin SJ. Use of antibiotics in preventing recurrent acute otitis media and in treating otitis media with effusion. A meta-analytic attempt to resolve the brouhaha. JAMA 1993;270:1344-51.

9 Gates GA, Avery CA, Prihoda TJ, Cooper JC. Effectiveness of adenoidectomy and tympanostomy tubes in the treatment of chronic otitis media with effusion. New Engl J Med 1987;3:1444-51

10 Maw AR, Herod F. Otoscopic, impedance and audiometric findings in glue ear treated by adenoidectomy and tonsillectomy. Lancet 1986;1:1399-402.

11 Paradise JL, Bluestone CD, Colborn DK, Bernard BS, Smith CG, Rockettte HE, et al. Adenoidectomy and adenotonsillectomy for recurrent acute otitis media. Parallel ranAdenoidectomy and adenotonsilectomy for recurrent acute otitis media. Parallel ran-
domized clinical trials in children not previously treated with tympanostomy tubes. domized clinical trials

12 Paradise JL, Bluestone CD, Rogers KD, Taylor FH, Colborn DK, Bachman RZ, et al. Efficacy of adenoidectomy for recurrent otitis media in children previously treated with tympanostomy-tube placement. Results of parallel randomized and nonrandomized trials. JAMA 1990;263:2066-73.

13 Alho O-P, Koivunen P, Luotonen J. Diagnostic criteria for otitis media in children. Otorhinolaryngol Nova 1999; 8:123-8.

14 Alho O-P, Läärä E, Oja H. What is the natural history of recurrent acute otitis media in infancy? J Fam Pract 1996;43:258-64.

15 Brown RA, Swanson Beck J. Medical statistics on personal computers. London: BMJ, 1994 16 Gardner MJ, Altman DG. Statistics with confidence. London: BMJ Publishing, 1989.

17 Bluestone CD. Role of surgery in the era of resistant bacteria. Pediatr Infect Dis J Bluestone CD. Rol
1998;17:1090-8.

18 Mattila P, Joki-Erkkilä VP, Kilpi T, Jokinen J, Herva E, Puhakka H. Prevention of otitis media by adenoidectomy in children younger than 2 years. Arch Otolaryngol Head Neck Surg 2003;129:163-8. 
19 Coyte PC, Croxford R, McIsaac W, Feldman W, Friedberg J. The role of adjuvant adenoidectomy and tonsillectomy in the outcome of the insertion of tympanostomy tubes. N Engl J Med 2001;344:1188-95.

20 Alho O-P, Koivu M, Sorri M, Rantakallio P. The occurrence of acute otitis media in infants-a life table analysis. Int J Pediatr Otorhinolaryngol 1991;21:7-14.

(Accepted 27 November 2003)

doi $10.1136 / \mathrm{bmj} .37972 .678345 .0 \mathrm{D}$
University of Oulu, PO Box 5000, FIN-90014, Finland

Petri Koivunen comsultant in otolaryngology

Matti Uhari professor of paediatrics

Jukka Luotonen consultant in otolaryngology

Aila Kristo specialist in otolaryngology

Risto Raski specialist in otolaryngology

Tytti Pokka statistician

Olli-Pekka Alho senior lecturer in otolaryngology

Correspondence to: Petri Koivunen petri.koivunen@ppshp.fi

$\stackrel{\infty}{3}$ 